\title{
Effects of aerobic exercise on antioxidants in rat models with cardiomyopathy
}

\author{
Eun-Jung Kima, Sujin Hwang ${ }^{\mathrm{b}}$ \\ ${ }^{a}$ Department of Physical Therapy, Dongshin University, Naju, Republic of Korea \\ ${ }^{b}$ Department of Physical Therapy, Baekseok University, Cheonan, Republic of Korea
}

Objective: In this study, we aimed to test the hypothesis that aerobic exercise might exert its cardio-protective effect by preventing oxidative stress and improving cardiac function in rat models with doxorubicin-induced cardiomyopathy.

Design: Randomized controlled trial.

Methods: We randomly divided experimental rats into four groups: the normal group was used as a non-cardiomyopathy normal control $(n=10)$; the control group included non-aerobic exercise after doxorubicin-induced cardiomyopathy $(n=10)$; the experimental group I included aerobic exercise $(3 \mathrm{~m} / \mathrm{min})$ after doxorubicin-induced cardiomyopathy $(\mathrm{n}=10)$; and experimental group II included aerobic exercise $(8 \mathrm{~m} / \mathrm{min})$ after doxorubicin-induced cardiomyopathy. Rats in the treadmill training groups underwent treadmill training, which began at 2 weeks after the first intraperitoneal injection. At the end of the exercise period, we determined the heart weight change for each rat. Changes in the levels of oxidative stress enzymes (superoxide dismutase [SOD], thiobarbituric acid-reactive substances [TBARS], and catalase) in the cardiac tissue of rats from all four groups were examined at the end of the experiment.

Results: Significant cardiac myocyte injury and increase in myocardial TBARS concomitant with a reduction in myocardial SOD and catalase were observed following cardiomyopathy $(p<0.05)$. Significant cardiac tissue and increase in myocardial TBARS along with reduction in myocardial SOD and catalase were observed following cardiomyopathy $(p<0.05)$. Oxidative parameters were significantly improved in the aerobic exercise groups compared with the control group.

Conclusions: These findings indicate that aerobic exercise effectively prevents oxidative stress in rat models with cardiomyopathy.

Key Words: Cardiomyopathies, Exercise, Oxidative stress

\section{Introduction}

Cardiomyopathy related to disease of the heart muscle has several causes, including obesity, excessive alcohol intake, inflammation, stress, nutritional deficiencies, genetic disorder, and post-childbirth [1]. Many pathological processes affect muscles and cause a loss of functional cardiac muscle cells. In cardiomyopathy, the heart muscle becomes enlarged, thick, or rigid $[2,3]$. As cardiomyopathy worsens, the heart becomes weaker. Oxidative stress plays a major role in the biochemical and pathophysiological changes associated with cardiomyopathy. Cardiomyopathy has been shown to be associated with increased oxidative stress, as evidenced by an increase in myocardial thiobarbituric acid-reactive substances (TBARS) and depletion of myocardial endogenous antioxidants such as C-reactive protein, superoxide dismutase (SOD), catalase, glutathione, and glutathione peroxidase [4-6]. Similar observations have been made previously in different studies using similar models [7,8]. Cardiac rehabilitation focuses on relieving symptoms and improving function. This may include exercise training and diet supplementation as methods for regulating oxidative

Received: 7 May, 2015 Revised: 1 June, 2015 Accepted: 10 June, 2015

Corresponding author: Eun-Jung Kim

Department of Physical Therapy, Dongshin University, 185 Geonjae-ro, Naju 520-714, Republic of Korea

Tel: 82-61-330-3390 Fax: 82-61-330-3394 E-mail: ddosuny@hanmail.net

(c) This is an Open-Access article distributed under the terms of the Creative Commons Attribution Non-Commercial License (http://creativecommons.org/licens es/by-nc/4.0) which permits unrestricted non-commercial use, distribution, and reproduction in any medium, provided the original work is properly cited.

Copyright $@ 2015$ Korean Academy of Physical Therapy Rehabilitation Science 
stress [9]. Gielen et al. [10] reported that exercise training improves cardiovascular function in individuals with cardiovascular disease. Exercise has beneficial effects on their activities of daily living, health-related quality of life, and ultimately their hospital admission rate and mortality for cardiovascular disease [11-14]. Doxorubicin, an anthracycline antibiotic, is widely used as an effective antineoplastic agent in the treatment of a variety of malignancies, including leukemia, lymphoma, and solid tumors [15]. Unfortunately, the clinical use of this drug is limited by cumulative dose-related cardiotoxicity, which may lead to a severe and irreversible form of cardiomyopathy [16]. Doxorubicin used to treat cancer has the serious side effect of dilated cardiomyopathy $[17,18]$. In view of this observation, the present study was designed to investigate whether aerobic exercise could offer protection against oxidative stress arising in a rat model out of cardiomyopathy.

\section{Methods}

\section{Cardiomyopathy rat model}

Forty 6-week-old male Sprague-Dawley rats, weighing $120.0 \pm 5.0 \mathrm{~g}$ were used following a 1-week acclimatization period. The rats were housed at a temperature of $25.0^{\circ} \mathrm{C} \pm$ $1.0^{\circ} \mathrm{C}$ and a humidity level of $55 \pm 2 \%$ with a 12 -h light-dark cycle; they had free access to food and water. All animal experimental protocols were performed in accordance with the guidelines of the Dongshin University Animal Care and Use Committee. We randomly divided experimental rats into four groups: the normal group was used as a non-cardiomyopathy normal control $(n=10)$; the control group included non-aerobic exercise after doxorubicin-induced cardiomyopathy $(\mathrm{n}=10)$; the experimental group I included aerobic exercise ( $3 \mathrm{~m} / \mathrm{min}$ ) after doxorubicin-induced cardiomyopathy $(\mathrm{n}=10)$; and experimental group II included aerobic exercise $(8 \mathrm{~m} / \mathrm{min})$ after doxorubicin-induced cardiomyo- pathy.

\section{Aerobic exercise and biochemical analysis}

Doxorubicin $(10 \mathrm{mg} / \mathrm{kg}$, Sigma-Aldrich Co., St. Louis, NY, USA) was dissolved in normal saline. The doses of doxorubicin used in this study were based previous reports [19]. Rats in the treadmill training group underwent treadmill training, which began at 2 weeks after first intraperitoneal injection. Treadmill exercise was performed according to a previously described method [20]. The treadmill velocity was set at $3 \mathrm{~m} / \mathrm{min}$ (experimental group I) and $8 \mathrm{~m} / \mathrm{min}$ (experimental group II) at a $0^{\circ}$ degree incline, and treadmill exercise was performed by rats over a 21-day period. Rats in the control group were allowed to move freely in their cages, but no additional treadmill running was employed. We determined the heart weight change for each rat after the 21 days of exercise. TBARS measured as a marker of lipid peroxidation and endogenous antioxidants, e.g., SOD and catalase [21-23].

\section{Data analysis}

Data analysis was performed using IBM SPSS Statistics for Windows ver. 21.0 (IBM Co., Armonk, NY, USA). All the data are expressed as mean (standard deviation) of 3 replications. Differences between two groups were tested by one-way ANOVA, followed by the Student-Newman- Keuls multiple comparisons test when difference were detected. $p$-value less than 0.05 at $95 \%$ confidence level was considered significant.

\section{Results}

As shown in Table 1, heart weight decreased significantly rat models of doxorubicin-induced cardiomyopathy compared to the control group $(p<0.05)$. The effects of aerobic exercise on the oxidative stress-related physiological factors

Table 1. Rat body weight and heart weight

\begin{tabular}{lrccc}
\hline Variable $(\mathrm{g})$ & Normal group & Control group & Experimental group I & Experimental group II \\
\hline Body weight & $266.00(4.58)$ & $238.22(15.04)^{\mathrm{a}}$ & $275.00(13.29)^{\mathrm{b}}$ & $257.50(36.59)^{\mathrm{b}}$ \\
Heart weight & $1.17(0.12)$ & $0.95(0.08)^{\mathrm{a}}$ & $1.15(0.05)$ & $1.00(0.04)$ \\
Weight ratio & $0.44(0.01)$ & $0.40(0.02)$ & $0.42(0.02)$ & $0.39(0.01)$ \\
\hline
\end{tabular}

Values are presented as mean (SD).

Normal group: normal rat, control group: non-treatment after doxorubicin-induced cardiotoxicity, experimental group I: low-intensity treadmill training $(3 \mathrm{~m} / \mathrm{min})$ after doxorubicin-induced cardiotoxicity, experimental group II: low-intensity treadmill training $(8 \mathrm{~m} / \mathrm{min})$ after doxorubicin-induced cardiotoxicity.

Tested by one-way ANOVA; ${ }^{\mathrm{a}} p<0.05$ compared with normal group, ${ }^{\mathrm{b}} p<0.05$ compared with control group. 


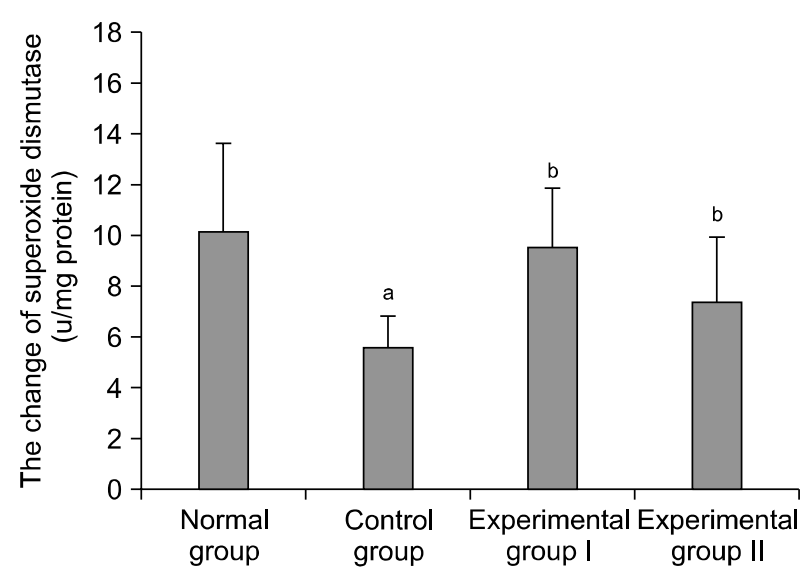

Figure 1. The effect of aerobic exercise on superoxide dismutase in cardiomyopathy rat model. Values are presented as mean (SD). Normal group: normal rat, control group: non-treatment after doxorubicin-induced cardiomyopathy, experimental group I: aerobic exercise $(3 \mathrm{~m} / \mathrm{min})$ after doxorubicin-induced cardiomyopathy, experimental group II: aerobic exercise $(8 \mathrm{~m} / \mathrm{min})$ after doxorubicin-induced cardiomyopathy. Tested by one-way ANOVA; ${ }^{\mathrm{a}} p<$ 0.05 compared with normal group, ${ }^{\mathrm{b}} p<0.05$ compared with control group.

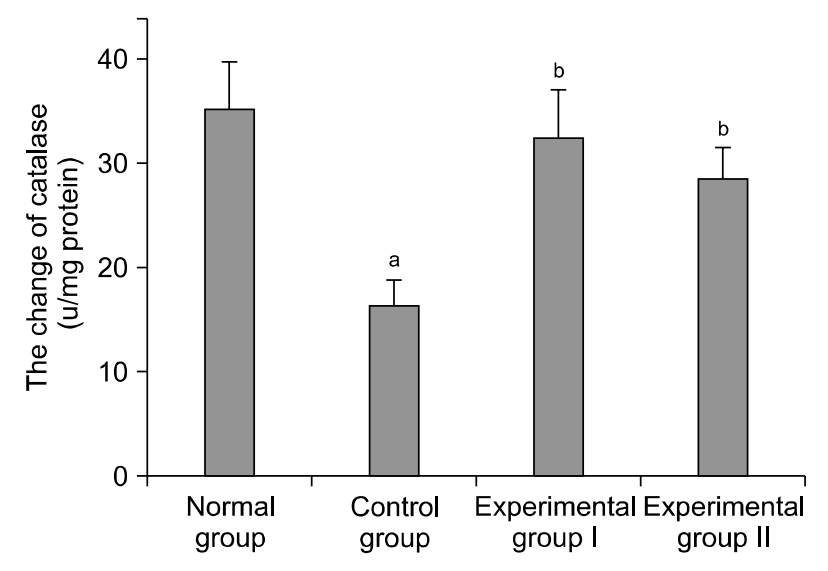

Figure 2. The change of aerobic exercise on thiobarbituric acid reactive substances in cardiomyopathy rat model. Values are presented as mean (SD). Normal group: normal rat, control group: non-treatment after doxorubicin-induced cardiomyopathy, experimental group I: aerobic exercise $(3 \mathrm{~m} / \mathrm{min})$ after doxorubicin-induced cardiomyopathy, experimental group II: aerobic exercise $(8 \mathrm{~m} / \mathrm{min})$ after doxorubicin-induced cardiomyopathy. Tested by one-way ANOVA; ${ }^{a} p<0.05$ compared with normal group, ${ }^{\mathrm{b}} p<0.05$ compared with control group.

in rats with caromyopathy are shown in Figures 1-3. In the aerobic exercise groups, oxidative stress factors related to heart muscle (e.g., SOD and catalase) were significantly increased after 21 days of aerobic exercise (Figures 1,2), and there was a in TBARS decrease $(p<0.05)$ (Figure 3$)$. There

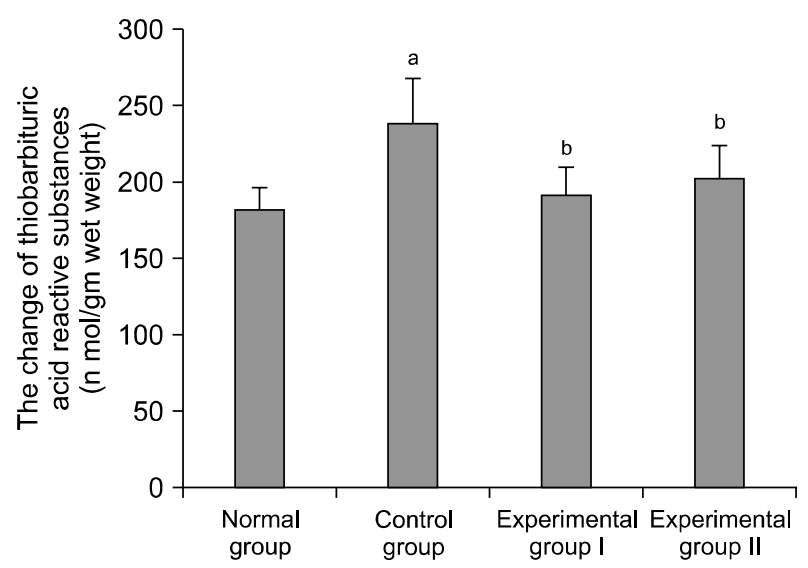

Figure 3. The change of aerobic exercise on catalase in cardiomyopathy rat model. Values are presented as mean (SD). Normal group: normal rat, control group: non-treatment after doxorubicin-induced cardiomyopathy, experimental group I: aerobic exercise $(3 \mathrm{~m} / \mathrm{min})$ after doxorubicin-induced cardiomyopathy, experimental group II: aerobic exercise $(8 \mathrm{~m} / \mathrm{min})$ after doxorubicin-induced cardiomyopathy. Tested by one-way ANOVA; ${ }^{\mathrm{a}} p<$ 0.05 compared with normal group, ${ }^{\mathrm{b}} p<0.05$ compared with control group.

was, however, no difference between the experimental groups.

\section{Discussion}

Cardiomyopathy can lead to an inability of the heart to effectively circulate blood through the body a state known as heart failure [24]. Physical activity is one of the core components in cardiac rehabilitation and secondary complication prevention [25]. While diverse approaches have been adopted for treating cardiomyopathy, the therapeutic efficacy and mechanism of action of the ideal approach have yet to be elucidated, although many studies are currently investigating this. Thus, the purpose of this study was to evaluate the effect of aerobic exercise training on cardiomyopathy in rats with doxorubicin-induced conditions as an exercise intervention for reducing oxidants within the muscle tissues.

Doxorubicin acts by forming an iron-anthracycline complex that generates free radicals, which in turn, causes severe damage to the muscle cell membrane, and interferes with cytoskeletal structure [26]. The decrease in body weight and heart weight in this study is in accordance with other studies [27] and it may be attributed to reduced dietary antioxidant intake and inhibition of protein synthesis due to doxorubicin treatment compared to the normal group. Oxygen free radical formation mediated by doxorubicin enhances the susceptibility of cardiac tissue to lipid peroxidation leading to a 
progressive dose-related irreversible loss of myofibrils, dilation of the sarcoplasmic reticulum, cytoplasmic vacuolization, swelling of mitochondria, increased number of lysosomes, and myocyte necrosis [28]. Matysiak et al. [29] reported increases in superoxide dysmutase- 1 and glutathione peroxidase concentrations during regular physical exercise, the latter of which was significantly suppressed by interval cycloergometer training.

Therapeutic strategies, designed to augment cellular endogenous defense systems such as antioxidants have been identified as a promising approach to combat doxorubicin toxicity [30]. According to our study, the oxidant enzyme concentrations in cardiac muscle were consistently reliably different in each experimental group and depended on aerobic exercise. In the rats with induced cardiomyopathy, TBARS levels were highest when compared with the normal group. Myocardial SOD and catalase activities were significantly decreased compared with the control group, and were correlated with cell death and severity of left ventricular dysfunction [31]. Oxidative stress plays a central role in the etiopathogenesis of cardiac dysfunction, and protection against oxidative stress through a cellular mechanism such as like myocardial adaptation holds promise as an effective therapeutic approach. Myocardial adaptation against oxidative stress is mediated through augmentation of a number of cellular antioxidants, such as SOD, catalase, glutathione peroxidase, and glutathione [32]. We have also reported that low-intensity exercise causes a significant decrease in basal glutathione and lactate dehydrogenase activities in rat serum, which is associated with a concomitant decrease in myocarditis [33]. Oxidative stress in cardiac tissue is caused by histological changes in cardiomyocyte size. A marked increase in cardiomyocyte apoptosis and concomitant induction of myocardial fibrosis was observed. Increased extracellular matrix content contributes to diastolic stiffness, and ultimately promotes ventricular dysfunction [34]. Reduction in oxidative stress through aerobic exercise will lead to an improvement in functional changes. Thus, aerobic exercise would be a major contributor to improved cardiac function in doxorubicin-induced cardiomyopathy.

The results of this study cannot be generalized for human population, because the design of this study was a rat models with cardiomyopathy. Further study will conduct for human population with or without cardiopulmonary dysfunction to evaluate the effect of aerobic exercise on cardiac functions in persons with or without cardiopulmonary dysfunction. In light of these results, aerobic exercise may play a role in decreasing TBARS and in increasing SOD and catalase. The findings of this study using animal models suggest that aerobic exercise as a therapeutic intervention can increase cardiac muscle antioxidant enzyme levels and reduce the levels of oxidative enzymes, which can suppress tissue inflammation and damage and promote heart function recovery.

\section{Conflict of Interest}

The authors declared no potential conflicts of interest with respect to the authorship and/or publication of this article.

\section{References}

1. Jefferies JL, Towbin JA. Dilated cardiomyopathy. Lancet 2010; 375:752-62.

2. McNally EM, Golbus JR, Puckelwartz MJ. Genetic mutations and mechanisms in dilated cardiomyopathy. J Clin Invest 2013; 123:19-26.

3. Maron BJ. Hypertrophic cardiomyopathy: a systematic review. JAMA 2002;287:1308-20.

4. Wojciechowska C, Romuk E, Tomasik A, Skrzep-Poloczek B, Nowalany-Kozielska E, Birkner E, et al. Oxidative stress markers and C-reactive protein are related to severity of heart failure in patients with dilated cardiomyopathy. Mediators Inflamm 2014;2014:147040.

5. Zhang L, Ma J, Liu H. Protective effect of ischemic postconditioning against ischemia reperfusion-induced myocardium oxidative injury in IR rats. Molecules 2012;17:3805-17.

6. Ilkun O, Boudina S. Cardiac dysfunction and oxidative stress in the metabolic syndrome: an update on antioxidant therapies. Curr Pharm Des 2013;19:4806-17.

7. Kaul N, Siveski-Iliskovic N, Hill M, Khaper N, Seneviratne C, Singal PK. Probucol treatment reverses antioxidant and functional deficit in diabetic cardiomyopathy. Mol Cell Biochem 1996;160-161:283-8.

8. Rodrigues B, McNeill JH. The diabetic heart: metabolic causes for the development of a cardiomyopathy. Cardiovasc Res 1992; 26:913-22.

9. Gąsiorowski A, Dutkiewicz J. Comprehensive rehabilitation in chronic heart failure. Ann Agric Environ Med 2013;20:606-12.

10. Gielen S, Schuler G, Adams V. Cardiovascular effects of exercise training: molecular mechanisms. Circulation 2010;122:1221-38.

11. Working Group on Cardiac Rehabilitation and Exercice Physiology and Working Group on Heart Failure of the European Society of Cardiology. Recommendations for exercise training in chronic heart failure patients. Eur Heart J 2001;22:125-35.

12. Wilson MG, Ellison GM, Cable NT. Basic science behind the cardiovascular benefits of exercise. Heart 2015;101:758-65.

13. Powers SK, Sollanek KJ, Wiggs MP, Demirel HA, Smuder AJ. Exercise-induced improvements in myocardial antioxidant capacity: the antioxidant players and cardioprotection. Free Radic 
Res 2014;48:43-51.

14. Rivera-Brown AM, Frontera WR. Principles of exercise physiology: responses to acute exercise and long-term adaptations to training. PM R 2012;4:797-804.

15. Ogura M. Adriamycin (doxorubicin). Gan To Kagaku Ryoho 2001;28:1331-8.

16. Dunn J. Doxorubicin-induced cardiomyopathy. J Pediatr Oncol Nurs 1994;11:152-60.

17. Heger Z, Cernei N, Kudr J, Gumulec J, Blazkova I, Zitka O, et al. A novel insight into the cardiotoxicity of antineoplastic drug doxorubicin. Int J Mol Sci 2013;14:21629-46.

18. Singal PK, Iliskovic N. Doxorubicin-induced cardiomyopathy. N Engl J Med 1998;339:900-5.

19. Fouad AA, Albuali WH, Al-Mulhim AS, Jresat I. Cardioprotective effect of cannabidiol in rats exposed to doxorubicin toxicity. Environ Toxicol Pharmacol 2013;36:347-57.

20. Albeck DS, Sano K, Prewitt GE, Dalton L. Mild forced treadmill exercise enhances spatial learning in the aged rat. Behav Brain Res 2006;168:345-8.

21. Ohkawa H, Ohishi N, Yagi K. Assay for lipid peroxides in animal tissues by thiobarbituric acid reaction. Anal Biochem 1979;95: 351-8.

22. Kakkar P, Das B, Viswanathan PN. A modified spectrophotometric assay of superoxide dismutase. Indian J Biochem Biophys 1984;21:130-2.

23. Aebi H. Catalase. In: Bergmeyer HU, ed. Methods of enzymatic analysis. 2nd ed. Weinheim: Chemic Academic Press Inc., Verlag; 1974. p. 673-85.

24. Rubler S, Dlugash J, Yuceoglu YZ, Kumral T, Branwood AW, Grishman A. New type of cardiomyopathy associated with diabetic glomerulosclerosis. Am J Cardiol 1972;30:595-602.

25. Chicco AJ, McCune SA, Emter CA, Sparagna GC, Rees ML, Bolden DA, et al. Low-intensity exercise training delays heart failure and improves survival in female hypertensive heart fail- ure rats. Hypertension 2008;51:1096-102.

26. Billingham ME, Bristow MR, Glatstein E, Mason JW, Masek MA, Daniels JR. Adriamycin cardiotoxicity: endomyocardial biopsy evidence of enhancement by irradiation. Am J Surg Pathol 1977;1:17-23.

27. Alimoradi H, Barzegar-Fallah A, Hassanzadeh G, MohammadiRick S, Asadi F, Delfan B, et al. The cardioprotective effects of an antiemetic drug, tropisetron, on cardiomyopathy related to doxorubicin. Cardiovasc Toxicol 2012;12:318-25.

28. Minotti G, Menna P, Salvatorelli E, Cairo G, Gianni L. Anthracyclines: molecular advances and pharmacologic developments in antitumor activity and cardiotoxicity. Pharmacol Rev 2004;56: 185-229.

29. Matysiak R, Błaszczyk J, Obrebska A, Mejer A, Koziróg M, Kowalski J. Oxidative-reductive balance in patients after acute coronary syndrome that undergo cardiac rehabilitation. Pol Merkur Lekarski 2014;37:148-52.

30. Steare SE, Yellon DM. The potential for endogenous myocardial antioxidants to protect the myocardium against ischaemia-reperfusion injury: refreshing the parts exogenous antioxidants cannot reach? J Mol Cell Cardiol 1995;27:65-74.

31. Frustaci A, Russo MA, Morgante E, Scopelliti F, Aquilano K, Ciriolo MR, et al. Oxidative myocardial damage in human cocaine-related cardiomyopathy. Eur J Heart Fail 2015;17:283-90.

32. Schaefer J, Nierhaus KH, Lohff B, Peters T, Schaefer T, Vos R. Mechanisms of autoprotection and the role of stress-proteins in natural defenses, autoprotection, and salutogenesis. Med Hypotheses 1998;51:153-63.

33. Ki YK, Kim GY, Kim EJ. Effects of cardiac biological activities on low-intensity physical training in doxorubicin-induced cardiotoxicity rat models. J Phys Ther Rehab Sci 2014;3:107-11.

34. Swynghedauw B. Molecular mechanisms of myocardial remodeling. Physiol Rev 1999;79:215-62. 